\title{
Bilateral Germ cell tumours in a patient with Swyer Syndrome
}

\author{
M Weerakkody ${ }^{1} \mathbf{R}$ Warusahennadi ${ }^{2}$ A Jayawardena ${ }^{3} \mathbf{P}$ Amarathunga ${ }^{4}$ \\ ${ }^{1}$ Department of Endocrinology, Teaching Hospital Kurunegala. \\ 2Department of Haematology, Teaching Hospital Kurunegala. \\ ${ }^{3}$ Department of Obstetrics and Gynaecology, Faculty of Medicine, University of Colombo. \\ ${ }^{4}$ Department of Pathology, Faculty of Medicine, University of Colombo.
}

\section{Abstract}

Swyer syndrome, or pure gonadal dysgenesis is a medical condition associated with 46 XY karyotype and primary amenorrhoea in a phenotypic female. In this syndrome, there is an abnormality in testicular differentiation. Patients with disorders in sexual differentiation have an increased risk for development of gonadal malignancies. We report a case of a 14-year-old girl who was investigated for primary amenorrhoea, who was finally diagnosed with Swyer syndrome. At gonadectomy, this girl was found to have bilateral gonadoblastomas with right side being transformed into a germinoma. The case was complicated by the fact that the girl was treated for dyserythropetic anaemia, which may cause a hypo gonadotrophic hypogonadism, contributing to delayed puberty.

Received: 24th February 2018

Accepted revised version: $5^{\text {th }}$ March 2018

Published: 9th April 2018

DOI: http://doi.org/10.4038/sjdem.v8i1.7351

Correspondence e-mail: mudithaweerakkody@gmail.com

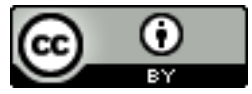

This is an open-access article distributed under the terms of the Creative Commons Attribution 4.0 International License, which permits unrestricted use, distribution and reproduction in any medium provided the original author and source are credited 


\section{Introduction}

Gonadal dysgenesis is a term used to describe a subset of disorders that are characterized by incomplete development of ovaries or testes, due to defects in sex chromosomes or mutations in genes involved with the development of gonads (1). In complete gonadal dysgenesis no gonadal development occurs, therefore the patients have a female phenotype due to the lack of gonadal steroids. These children are reared as females and the problem comes to light when they present with delayed puberty. Patients with gonadal dysgenesis who have a Y chromosome or Y-chromosome material are at increased risk for developing germ cell tumors such as gonadoblastoma or carcinoma in situ, with the potential for malignant transformation to dysgerminoma or seminoma, respectively (2).

We describe a case of a 14-year-old girl, on follow up for dyserythropetic anemia, who was referred for further evaluation of delayed puberty. Both hypo gonadotrophic hypogonadism and hyper gonadotrophic hypogonadism can occur due to iron overload conditions, due to iron deposition in pituitary gland and gonads respectively. Although hypo gonadotrophic hypogonadism is what we commonly find in such cases, this girl had very high gonadotrophin levels. Therefore, we undertook extensive evaluation of this girl, which led us to the final diagnosis of complete gonadal dysgenesis. The issue was complicated by the fact that one of the dysgenetic gonads was in the process of malignant transformation.

\section{Case}

A 14-year-old girl, an only child of a non-consanguineous marriage, was referred to us by the haematology unit for further investigation of primary amenorrhea. She was on follow up at the hematology unit for a congenital dyserythropoetic anaemia but was not transfusion dependent. There were no other significant medical illnesses or comorbidities. The mother attained menarche at the age of 13 years, and there was no family history of delayed puberty. On examination, she was an averagely built girl with height and weight both on the $25^{\text {th }}$ centile on the WHO (World Health Organization) growth chart. There were no features of dysmorphism, and the systems examination didn't reveal any abnormality. She also featured breast development of Tanner stage 1, Tanner stage 1 pubic hair with no axillary hair development.
The patient exhibited female external genitalia, with normal labia majora and minora and a visible vaginal orifice. No palpable masses were identified in the groin or labia majora. Laboratory analyses showed: FSH 55.8 $\mathrm{mIU} / \mathrm{ml}$ (1.4-11.7), LH $22 \mathrm{mIU} / \mathrm{ml}$ (0.6-21), estradiol 13.07 (13-71) pg/ml, total testosterone $0.2 \mathrm{ng} / \mathrm{ml}(0-0.73$ $\mathrm{ng})$, cortisol $10.1 \mu \mathrm{g} / \mathrm{dl}(8.7-25)$, free T4 $0.91 \mathrm{ng} / \mathrm{ml}(0.93$ -

1.7) $\mathrm{TSH} 1.9 \mathrm{micIU} / \mathrm{ml}(0.55-4.78)$ and prolactin 8.25 $\mathrm{ng} / \mathrm{ml}(3.9-29.5)$.

Her serum ferritin level was low at $6.38 \mathrm{ng} / \mathrm{ml}$. Although, due to the dyserythropetic anemia associated with iron overload, we expected to find a hypo gonadotrophic hypogonadism with low FSH and LH levels, this girl had elevated gonadotrophin levels, which led us to consider a different etiology for her delayed puberty.

Ultrasound scan abdomen revealed splenomegaly and a small uterus, but bilateral ovaries were not separately identified. Although the next investigation of choice is magnetic resonance imaging (MRI) (2), we first had to perform a contrast enhanced computer tomography (CT), as MRI is not freely available in our setting. Both CT scanning and MRI demonstrated a rudimentary uterus with absent gonads. The patients' karyotype was revealed as $46 \mathrm{XY}$ and she was found to be positive for SRY gene.

As the patient was reared as a girl from birth, after discussing with both the parents and the child, it was decided that she would go ahead with laparoscopy and gonadectomy with subsequent introduction of hormone replacement to develop and mature female secondary sexual characteristics. At laparoscopy, right sided fallopian tube and gonad were found to be enlarged and the left side gonad was small and streaky. Bilateral gonads and fallopian tubes were removed and sent for histology. Histologically, the right sided gonadal appearance was keeping with a gonadoblastoma (figure 1) with areas suspicious of transforming into a germinoma (figure 2).

Immunohistochemical analysis demonstrated strong membrane positivity for CD117, D2-40 and PLAP. The left side gonad also demonstrated morphological features of a gonadoblastoma. The patient was subsequently referred for chemotherapy to National Cancer Institute, Maharagama. She would have continuous follow up at the Endocrine unit and would be commenced on hormone replacement for pubertal induction, once the chemotherapy is completed. 


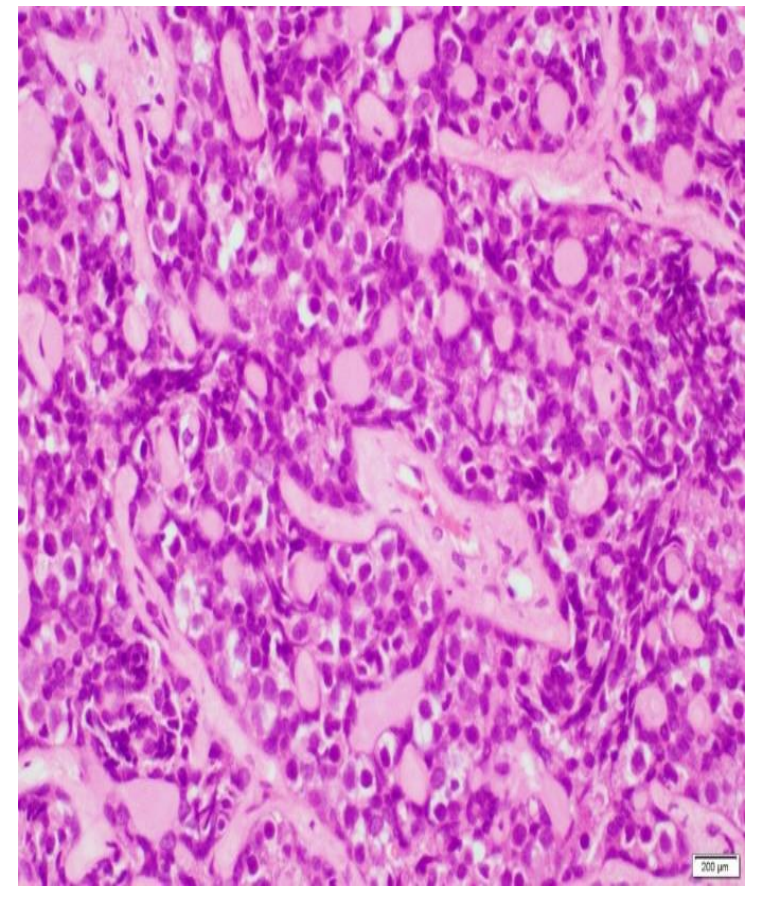

Figure 1: Gonadoblastoma showing primitive germ cells and sex cord stromal cells surrounded by eosinophilic globular basement membrane like material ( $\mathrm{H}$ and $\mathrm{E})$.

\section{Discussion}

The first reported case of Swyer syndrome was in 1955 by Dr G I Swyer who described two women who had a 46, XY karyotype, tall stature, primary amenorrhea, female external genitalia, and a normal vagina and cervix (3). Individuals with Swyer syndrome are phenotypically female with unambiguously female genitalia and hypoplastic to normal Müllerian structures. The patients first present in adolescence with delayed puberty and primary amenorrhea. As expected, they show elevated gonadotropins, normal female levels of androgens, and low levels of estrogens.

Although the genetic sex of an individual is determined at fertilization, it is at the embryonic stage that the sexual differentiation of the reproductive system begins. The primordial gonad is bipotential and can differentiate into a testis or an ovary, depending on the SRY gene located in the short arm of the Y-chromosome. The absence of SRY permits the bipotential gonad to differentiate into an ovary at the $8^{\text {th }}$ week of the embryo, leading to the

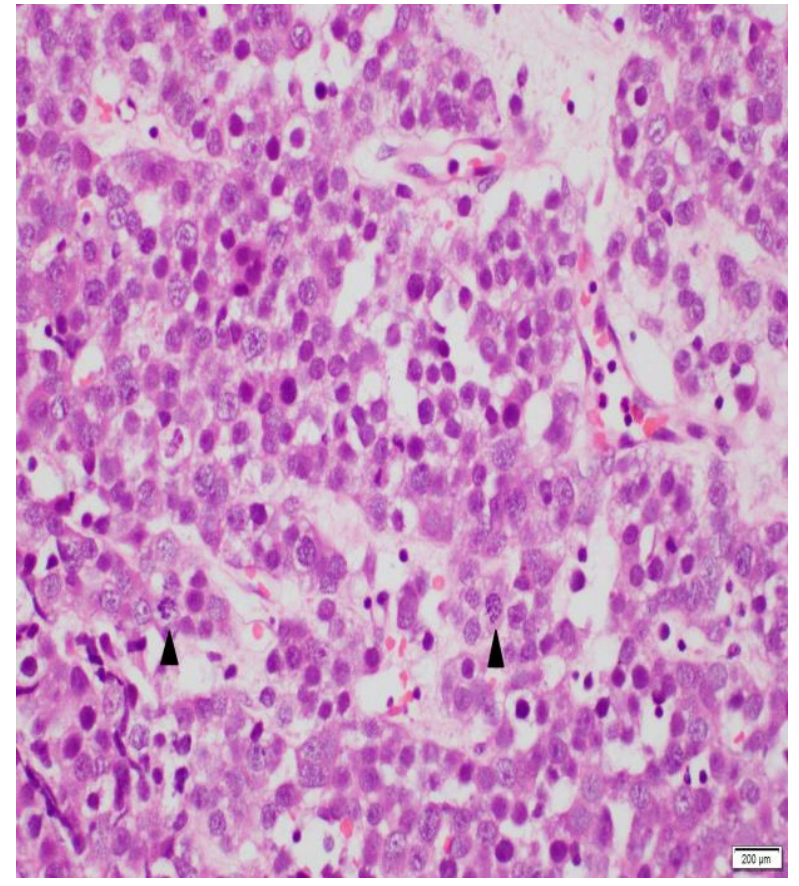

Figure 2: Germinoma component with primitive germ cells displaying regular nuclei with prominent nucleoli and clear cytoplasm. Mitotic figures are abundant (arrow heads) (H and $\mathrm{E}$ ).

female phenotype. The mutation, deletion or translocation of SRY can affect the binding of the SRY proteins with DNA, and consequently contribute to sex reversal (4). Therefore, SRY gene plays an important role in human testis development and its inactivating mutations will lead to the clinical picture seen in 46, XY complete gonadal dysgenesis. However, mutations in the SRY gene are found only in 10-20 \% of the cases (5). In our patient, as SRY gene was found in the chromosomal analysis, the possibility of another genetic mutation needs to be considered. Duplication of DAX1 (also known as NROB1) and WNT4 genes as well as haplo-insufficiency of the SOX9, SF1, WT1 and WMRT1—WMRT2 genes have also have been considered responsible for the development of 46, XY complete gonadal dysgenesis (4). These genes are involved in the regulation of SRY expression or act as a transcription activator downstream of SRY in the testis-determining pathway.

The risk of gonadal malignancy in complete gonadal dysgenesis is estimated to range from $37.5 \%-45 \%$ (2). 
Those patients with a gonadal malignancy, dysgerminoma was present in $22-66 \%$. The majority of cases of gonadoblastoma or dysgerminoma are discovered at the time the diagnosis of complete gonadal dysgenesis is established, typically in adolescence. However, there had been cases of malignancy in young children. The malignancy risk also rises with age and reaches $50-70 \%$ in the third decade and to around $80 \%$ by fifth decade (6). Therefore, the current recommendation is to arrange bilateral gonadectomy as soon as XY complete gonadal dysgenesis is diagnosed (2). However, this decision, as well as the diagnosis of $\mathrm{XY}$ complete gonadal dysgenesis itself, would have major psychosocial implications for the patient.
Therefore, the patient as well as the family would need multiple sessions of counselling regarding various aspects of the disease. After gonadectomy has been performed, exogenous hormone replacement therapy should be provided for XY female patients to initiate, mature, and maintain secondary sexual characteristics (6).

Cyclic estrogen and progestogen therapy initiated after the completion of estrogen-induced breast development will prevent endometrial hyperplasia that may result from unopposed estrogen stimulation (6). A multidisciplinary approach with the participation of the geneticist, endocrinologist, paediatrician, gynecologist and the psychologist would beneficial in managing these complex patients.

\section{References}

1. Lee PA, Houk CP, Ahmed SF: Consensus statement on management of intersex disorders: international consensus conference on intersex. Pediatrics 2006, 118: e488-e500

2. McCann-Crosby B, Mansouri R, Dietrich J et al. State of the art review in gonadal dysgenesis: challenges in diagnosis and management. International Journal of Pediatric Endocrinology 2014, 2014:4

3. Verp MS, Simpson JL: Abnormal sexual differentiation and neoplasia. Cancer Genet Cytogenet. 1987, 25:191-218.

4. Xue DU, Zhang X, Yongmeli Li, Yukun Han. 46, XY female sex reversal syndrome with bilateral gonadoblastoma and dysgerminoma. Exp Ther Med. 2014; 8:1102-4.

5. Thomas FG, King, Conway Gerard S. Swyer syndrome: a review. Reproductive Endocrinology. 2014; 21(6):504-10.

6. Keskin M, Savaş-Erdeve S, Kurnaz E et al. Gonadoblastoma in a patient with 46, XY complete gonadal dysgenesis. The Turkish Journal of Pediatrics 2016; 58: 538-540.

7. Jung Jung E, Hwa Im D, Park Y H et al. Female with 46, XY karyotype. Obstet Gynecol Sci 2017; 60(4):378-382. 